\title{
HORIZONTALE UND VERTIKALE DIFFERENZEN DER POLITISCHEN REPRÄSENTATION IN ÖSTERREICH
}

\author{
Carmen Walenta-Bergmann, Johannes Kepler Universität, Linz
}

\section{Zusammenfassung}

Das föderalistische politische System der demokratischen Republik Österreich ermöglicht durch Wahlen legitimierte Volksvertretung auf drei Ebenen: dem Bund, den Bundesländern und den Gemeinden. Basierend auf umfassenden Datensätzen geht dieser Beitrag der Frage nach, inwiefern sich die demokratisch legitimierten politischen RepräsentantInnen dieser drei politischen Ebenen in Bezug auf Parteizugehörigkeit und Geschlecht voneinander (vertikal) und innerhalb der substaatlichen Ebenen (horizontal) voneinander unterscheiden. Anhand der systematischen statistischen Auswertung von Daten der letzten 30 Jahre wird deutlich, dass es in Österreich anhaltende horizontale sowie vertikale Repräsentationsdifferenzen gibt. Als mögliche Gründe für die spezifisch österreichischen Differenzen werden unter anderem historische Ursachen diskutiert. Mögliche Auswirkungen der regionalen Repräsentationsdifferenzen hingegen sind manifeste subnationale Unterschiede des politischen Outcomes und Outputs in den von Gemeinden und Ländern dominierten Politikfeldern.

\section{Einleitung}

Wie in anderen föderal organisierten Ländern ist politische Repräsentation auch in Österreich mehrdimensional. Während sich in Einheitsstaaten die Repräsentationsstruktur über die Zeit hinweg einheitlich verändert, kann sich die Repräsentationsstruktur in Föderationen unterschiedlich schnell, oder sogar in entgegengesetzte Richtungen entwickeln. Diese aus der Logik von Mehrebenensystemen hervorgehende Tatsache, kann dazu führen, dass erhebliche Repräsentationsunterschiede zwischen der staatlichen und den substaatlichen Ebenen entstehen. Zusätzlich können sich auch die Repräsentationscharakteristika der substaatlichen Einheiten ein und derselben föderalen Ebene stark voneinander unterscheiden. Anhand des Fallbeispiels Österreich, geht dieser Beitrag der Frage nach ob und in welchem Ausmaß die Existenz solcher vertikalen und horizontalen Repräsentationsunterschiede innerhalbe eines Landes nicht nur theoretisch denkbar, sondern auch 
empirisch nachweisbar sind. Außerdem wird nach den Ursachen und Auswirkungen für existierende, beziehungsweise nichtexistierende vertikale und horizontale Repräsentationsunterschiede gesucht. Dieser Beitrag ist eine detaillierte Analyse der Partei- und Geschlechtsdimension der demokratisch legitimierten Volksvertretung in Österreich. Im Rahmen dieser Analyse werden die Ebene des Bundes, sowie die Ebene der neun Bundesländer und jene der aktuell 2095 Gemeinden miteinander verglichen. Anhand der systematischen statistischen Auswertung von fast 30 Jahren (sub)nationaler politischer Repräsentation wird deutlich, dass sich die nationale und subnationale politische Repräsentation in Österreich stark voneinander unterscheiden und die regionalen Differenzen sehr groß sind.

Nach dieser Einleitung wird ,Der österreichische Föderalismus' anhand von theoretischen und empirischen Analysen aus der vergleichenden Föderalismusforschung und der Multi-LevelGovernance-Forschung beschrieben und eingeordnet. Anschließend werden ,Forschungsfrage, Variablen und Hypothesen“ vorgestellt, gefolgt von der Beschreibung und Darstellung der ,Entwicklung der Politischen Repräsentation in den Bundesländern“ und der ,Entwicklung der Politischen Repräsentation in den Gemeinden؛. Schließlich werden ,Ursachen für und Auswirkungen von Repräsentationsdifferenzen“ besprochen, abgerundet durch eine abschließenden ,Zusammenfassung und Diskussion".

\section{Der österreichische Föderalismus}

Wie schon in der Einleitung angedeutet stellt Österreich ein interessantes Fallbeispiel für die Frage nach der Existenz und den Auswirkungen von innerstaatlichen politischen Repräsentationsdifferenzen dar. Österreich ist eine föderal organisierte, demokratische Republik. Es existieren demokratisch legitimierte Volksvertretung sowohl auf der Ebene des Bundes wie auch auf der Ebene der neun Bundesländer und den aktuell 2095 Gemeinden. Österreich ist im Jahr 2021 eine von nur 25 Föderationen weltweit. ${ }^{1}$ So sehr diese Fakten für die Relevanz des Falles Österreichs für die Analyse von horizontalen und vertikalen Repräsentationsdifferenzen sprechen, gibt es doch auch einige gewichtige Einwände. In der schon viele Jahrzehnte andauernden Diskussion über die Schwächen des spezifisch österreichischen Föderalismus werden immer wieder unitaristische Tendenzen konstatiert und sogar die Integrität des

1 Überblick zu Föderationen weltweit zu finden auf: http://www.forumfed.org/countries/ (,Forum of Federations. The global network on federalism and devolved governance") [last access: April 2021] 
föderalistischen Systems angezweifelt (vgl. z.B. Erk 2004). Die Gründe für solche Diagnosen sind vielfältig und reichen von der Entstehungsgeschichte des österreichischen Föderalismus, über Kompetenzverteilungen, bis hin zu fiskalischen Fragen. Die vergleichende Föderalismusforschung zeigt, dass vor allem im Vergleich mit anderen benachbarten Föderationen, wie der Schweiz oder Deutschland, dem österreichischen Föderalismus mindestens fünf entscheidende Schwächen zugeschrieben werden müssen. Die erste nimmt ihren Ausgang in der Entstehungsgeschichte der österreichischen Föderation. Diese war nicht wie beispielsweise eine bottom-up Entwicklung ausgehend von den Bundesländern, sondern eine top-down Entscheidung nach dem ersten Weltkrieg. Ein zweiter historischer Grund für die Schwäche des österreichischen Föderalismus ist die Tatsache, dass dieser von Anfang an nur eine Kompromisslösung zwischen der Christlichsozialen (heute ÖVP) und Sozialistischen Partei (heute SPÖ) war. Die Christlichsozialen befürworteten einen stark dezentralen Bundesstaat nach Vorbild der Schweiz, die Sozialistische Partei hingegen wollte ein einheitsstaatliches politisches System für den neuen österreichischen Staat (Bußjäger/Schramek 2020; Bischof/Karlhofer 2016; Kim/Mau 2015). Der dritte oft diskutierte Grund basiert auf der relativen Schwäche der zweiten Kammer des österreichischen Parlaments. Anders als in vielen anderen Ländern besitzt der Bundesrat lediglich ein Vetorecht, welches zusätzlich in der politischen Realität so gut wie nie geltend gemacht wird (Karlhofer 2013; Bischof/Karlhofer 2016; Fallend 2007). Eine vierte Schwäche sind die vergleichsweise geringen legislativen Kompetenzen auf subnationaler Ebene (Kim/Mau 2015; Behnke et al. 2019). Dadurch, dass Steuern primär vom Bund eingetrieben werden besitzt die substaatliche Ebene ist Österreich fünftens nur eine sehr geringe fiskalische Autonomie (Kim/Mau 2015).

Trotz dieser nicht in Abrede zu stellenden Schwächen kann man Österreich nicht als unitaristischen Staat bezeichnen. Einerseits, weil die administrativen Kompetenzen und die administrative Autonomie auf subnationaler Ebene sehr hoch sind (Fallend 2003; Karlhofer 2013). Andererseits aber auch, deswegen, weil die Schwäche des Bundesrates durch die Stärke der Landeshauptleutekonferenz kompensiert wird. Diese ist zwar nicht in der österreichischen Bundesverfassung vorgesehen, aber kann in der politischen Realität dennoch als wichtigstes Gremium der Länder bezeichnet werden (Bussjäger 2018). Drittens ist aus der Umfrageforschung bekannt, dass die Legitimität des föderalistischen Systems in der Bevölkerung fest verankert ist. Diese haben gegenüber den politischen Institutionen der Gemeinden und Bundesländer weitaus größeres Vertrauen als gegenüber den nationalstaatlichen 
(Karlhofer 2013). Diese Erkenntnisse aus der vergleichenden Föderalismusforschung können mit Ergebnissen aus der Multi-Level-Governance-Forschung ergänzt werden. In der MLG-Forschung versucht man nicht einzelne Föderationen miteinander zu vergleichen, sondern versucht die dynamische Verteilung von Macht auf die subnationale, nationale und supranationale Ebene zu verstehen (vgl. z.B. Bache/Flinders 2004; Enderlein et al. 2010). Um die Machtverteilung messen zu können wurden sogenannte Autorität- bzw. Autonomieindizes entwickelt. Sowohl die Messungen des RAI (Regional Authority Index), als auch jene des LAI (Local Autonomy Index) kommen zu dem Ergebnis, dass die lokale Autorität/Autonomie in Österreich in den letzten Jahrzehnten zugenommen hat (Hooghe et al. 2016; Ladner 2016). Zusammengefasst besitzt die subnationale Ebene in Österreich damit - sowohl aus Sicht der vergleichenden Föderalismus-, als auch aus Sicht der Multi-LevelGoverance-Forschung - ausreichend politischen Gestaltungsspielraum um für weiterführende Analysen (z.B. der Auswirkungen von Repräsentationsdifferenzen) relevant zu sein.

\section{Forschungsfrage, Variablen und Hypothesen}

Dieser Beitrag geht der Frage nach, inwiefern sich die demokratisch legitimierte politische Repräsentation der drei föderalen Ebenen in Österreich (Bund, Länder, Gemeinden) voneinander unterscheidet. Außerdem wird der Versuch unternommen die Entwicklung der Differenz über die Zeit hinweg abzubilden, einzuordnen und zu interpretieren. Dieses Forschungsvorhaben wird entlang zwei sehr zentraler Variablen empirisch umgesetzt: dem parteipolitischen Gleichgewicht und Geschlechtergleichgewicht der politischen Repräsentation.

Ob und inwiefern diese Repräsentationsvariablen überhaupt Einfluss auf Output und Outcome haben, und somit Relevanz für weiterführende Fragen besitzt, wird in der Politikwissenschaft seit vielen Jahrzehnten kontrovers diskutiert. Was den Einfluss der Parteipolitik betrifft versuchen Forscherlnnen spätestens seit den Arbeiten von Douglas Hibbs (1977) sowohl den Einfluss der Parteipolitik als auch deren Irrelevanz für Output und Outcome zu beweisen (Imbeau et al. 2001). Der ,New Politics of the Welfare State‘ Ansatz von Paul Pierson (1996; 2001) kommt zusätzlich zu dem Schluss, dass sozioökonomische Variablen viel besser geeignet sind um Unterschied in politischem Output und Outcome zu erklären als die Spezifika der politischen Repräsentation. 
Der Einfluss des Geschlechterverhältnisses, bzw. der Einfluss von Frauen in der politischen Repräsentation wird weit weniger kontrovers diskutiert. In der politikwissenschaftlichen Forschung ist man sich aktuell weitgehen darüber einig, dass die Veränderung der Geschlechterverhältnisse einen Einfluss auf das politische Output und Outcome haben. So kam man etwa im Bereich der Sozialpolitik zu dem Ergebnis, dass ein höherer Anteil an Frauen in der Repräsentation zu höheren wohlfahrtsstaatlichen Ausgaben führen (Bolzendahl/Brooks 2007; Bolzendahl 2011; Bonoli/Reber 2010). Dennoch sind die Auswirkungen des Geschlechterverhältnisses und die dahinterstehenden Mechanismen noch lange nicht in allen Einzelheiten geklärt. Die theoretischen Konzepte, welche für die Interpretation von empirischen Ergebnissen in diesem Bereich herangezogen werden können, reichen von der ,Critical Mass', über die ,Critical Actor' Theorie, bis hin zu Theorien, welche sich mit der Beziehung von deskriptiver und substantieller Repräsentation auseinandersetzten (vgl. z.B. Childs/Krook 2009).

Aus der Forschung im Bereich vertikaler und horizontaler Repräsentationsunterschiede in Demokratien ergeben sich einige interessante Schlussfolgerungen für die Variablen Partei und Geschlecht. Untersuchungen des National-Subnational Gender Gaps kamen etwa zu dem Ergebnis, dass die Repräsentationsquoten von Frauen auf nationaler Ebene am geringsten sind (vgl. z.B. EscobarLemmon/Funk 2018; Sundström/Stockemer 2015; Vengroff et al. 2003). Außerdem gibt es Evidenz dafür, dass das National-Subnational Gender Gap mit zunehmender Industrialisierung und wirtschaftlicher Entwicklung immer kleiner wird (Vengroff et al. 2003). Die Analyse der Kongruenz subnationaler Repräsentation zeigt, dass Österreich noch vor der Schweiz die geringsten horizontalen Unterschiede aufweist. Im Vergleich zu anderen untersuchten Ländern (z.B. Australien, Deutschland, USA) sind diese geringen Unterschiede über den Untersuchungszeitraum hinweg außerdem sehr stabil (Bolleyer/Bytzek 2009).

Aufgrund dieser Forschungsergebnisse können für Österreich vor allem drei Hypothesen aufgestellt werden: 1) Die vertikale Differenz der politischen Repräsentation ist gering, und wird über die Zeit hinweg immer kleiner. 2) Die horizontale Differenz der politischen Repräsentation ist niedrig und stabil. 3) Der Anteil an Frauen in der Repräsentation ist auf nationaler Ebene am geringsten. 


\section{Entwicklung der Politische Repräsentation in den Bundesländern}

Das politische System der neun österreichischen Bundesländer besteht wie jenes des Bundesstaates aus Legislative, Exekutive und Judikative. Die gesetzgebende Gewalt sind die Landtage mit jeweils 36 (Kärnten, Burgenland, Salzburg, Tirol, Vorarlberg), 48 (Steiermark), 56 (Niederösterreich, Oberösterreich), bzw. 100 Sitzen (Wien). Die vollziehende Gewalt ist die jeweilige Landesregierung (inkl. Landeshauptmann bzw. Landeshauptfrau) welche zwischen 7 und 15 Mitgliedern hat. Das Landesverwaltungsgericht schließlich ist die richterliche Gewalt (Dachs 2006).

Die Wahl der politischen Repräsentation in den österreichischen Bundesländern findet alle fünf bis sechs Jahre statt und wird durch das Wahlrecht der Landtage geregelt ${ }^{2}$. Die empirische Analyse der parteipolitischen Zusammensetzung der politischen Repräsentation zeigt eine Pluralisierung der Parteienlandschaft. Tabelle 1 zeigt die Entwicklung der Sitzanteile pro Partei in den Landtagen der österreichischen Bundesländer zwischen 1990 und 2020. Während im Jahr 1990 im Schnitt noch rund 85 Prozent der Sitze von ÖVP oder SPÖ Mitgliedern besetzt sind, sind es im Jahr 2020 nur mehr rund 65 Prozent.

Tabelle 1: Entwicklung der Sitzanteile pro Partei in den Landtagen der österreichischen Bundesländer

\begin{tabular}{lrrrrr} 
Jahr & Partei & Minimum & Mittelwert & Maximum & Standardabweichung \\
\hline OEVP & 1990 & 30.00 & 48.15 & 55.56 & 7.95 \\
OEVP & 2000 & 15.00 & 38.68 & 50.00 & 12.37 \\
OEVP & 2010 & 16.67 & 39.76 & 55.56 & 14.36 \\
OEVP & 2020 & 7.00 & 35.24 & 51.79 & 14.90 \\
\hline SPOE & 1990 & 22.22 & 36.85 & 62.00 & 12.99 \\
SPOE & 2000 & 13.89 & 32.36 & 47.22 & 10.13 \\
SPOE & 2010 & 8.33 & 32.87 & 55.00 & 16.07 \\
SPOE & 2020 & 11.11 & 29.40 & 52.78 & 15.35 \\
\hline FPOE & 1990 & 3.57 & 12.44 & 30.56 & 8.24 \\
FPOE & 2000 & 13.89 & 23.57 & 44.44 & 9.59 \\
FPOE & 2010 & 0.00 & 10.90 & 25.00 & 7.77 \\
FPOE & 2020 & 11.11 & 20.05 & 34.00 & 8.41 \\
\hline GRUE & 1990 & 0.00 & 2.56 & 8.33 & 3.26 \\
GRUE & 2000 & 0.00 & 4.33 & 8.33 & 2.87 \\
GRUE & 2010 & 2.78 & 7.95 & 14.00 & 3.59 \\
GRUE & 2020 & 0.00 & 9.22 & 19.44 & 5.43 \\
\hline NEOS & 1990 & 0.00 & 0.00 & 0.00 & 0.00 \\
NEOS & 2000 & 0.00 & 0.40 & 3.57 & 1.19
\end{tabular}

\footnotetext{
2 für detaillierte Informationen zum Wahlrecht der Landtage siehe: Ucakar et al. 2017, S.94ff und S.141ff
} 


\begin{tabular}{lrrrrr} 
NEOS & 2010 & 0.00 & 0.00 & 0.00 & 0.00 \\
NEOS & 2020 & 0.00 & 4.08 & 8.33 & 3.37 \\
\hline OTHE & 1990 & 0.00 & 0.00 & 0.00 & 0.00 \\
OTHE & 2000 & 0.00 & 0.67 & 6.00 & 2.00 \\
OTHE & 2010 & 0.00 & 8.51 & 47.22 & 15.87 \\
OTHE & 2020 & 0.00 & 2.01 & 8.33 & 3.19 \\
\hline
\end{tabular}

Quelle: Eigene Berechnungen auf Basis von Daten der Landtagshomepages der Bundesländer

Dieses Bild zeigt sich auch, wenn man die Sitzanteile pro Partei in den Landesregierungen betrachtet. Tabelle 2 gibt einen Überblick der Entwicklung der Sitzanteile pro Partei in den Landesregierungen der österreichischen Bundesländer. Hier ist der gemeinsame Sitzanteil von ÖVP und SPÖ zwischen 1990 und 2020 von rund 93 auf 75 Prozent gefallen.

Tabelle 2: Entwicklung der Sitzanteile pro Partei in den Landesregierungen der österreichischen Bundesländer

\begin{tabular}{lrrrrr} 
Partei & Jahr & Minimum & Mittelwert & Maximum & Standardabweichung \\
\hline OEVP & 1990 & 26.67 & 52.24 & 85.71 & 17.98 \\
OEVP & 2000 & 14.29 & 47.80 & 85.71 & 23.55 \\
OEVP & 2010 & 14.29 & 50.66 & 100.00 & 27.64 \\
OEVP & 2020 & 0.00 & 46.29 & 75.00 & 28.25 \\
\hline SPOE & 1990 & 0.00 & 40.87 & 66.67 & 20.33 \\
SPOE & 2000 & 0.00 & 35.36 & 50.00 & 14.87 \\
SPOE & 2010 & 0.00 & 36.90 & 64.29 & 22.14 \\
SPOE & 2020 & 0.00 & 28.87 & 71.43 & 29.27 \\
\hline FPOE & 1990 & 0.00 & 6.89 & 14.29 & 6.94 \\
FPOE & 2000 & 0.00 & 16.05 & 42.86 & 13.62 \\
FPOE & 2010 & 0.00 & 9.61 & 57.14 & 18.48 \\
FPOE & 2020 & 0.00 & 12.39 & 38.46 & 16.39 \\
\hline GRUE & 1990 & 0.00 & 0.00 & 0.00 & 0.00 \\
GRUE & 2000 & 0.00 & 0.79 & 7.14 & 2.38 \\
GRUE & 2010 & 0.00 & 2.82 & 14.29 & 5.66 \\
GRUE & 2020 & 0.00 & 9.63 & 28.57 & 11.14 \\
\hline NEOS & 1990 & 0.00 & 0.00 & 0.00 & 0.00 \\
NEOS & 2000 & 0.00 & 0.00 & 0.00 & 0.00 \\
NEOS & 2010 & 0.00 & 0.00 & 0.00 & 0.00 \\
NEOS & 2020 & 0.00 & 1.59 & 14.29 & 4.76 \\
\hline OTHE & 1990 & 0.00 & 0.00 & 0.00 & 0.00 \\
OTHE & 2000 & 0.00 & 0.00 & 0.00 & 0.00 \\
OTHE & 2010 & 0.00 & 0.00 & 0.00 & 0.00 \\
OTHE & 2020 & 0.00 & 2.09 & 11.11 & 4.23 \\
\hline OID & & & & \\
\hline
\end{tabular}

Quelle: Eigene Berechnungen auf Basis von Daten der Verwaltungshomepages der Bundesländer 
Dieser in Landtag und Landesregierung beobachtete Trend wird bei der Parteizugehörigkeit der Landeshauptleute noch nicht sichtbar. Tabelle 3 zeigt, dass der Anteil an ÖVP und SPÖ Landeshauptleuten ungebrochen hoch ist und im Jahr 2020 sogar bei 100 Prozent liegt.

Neben der Parteizugehörigkeit gibt Tabelle 3 auch einen Überblick über den Frauenanteil unter den Landeshauptleuten. Dieser hat sich über den Untersuchungszeitraum hinweg nicht erhöht. Lediglich unten den stellvertretenden Landeshauptleuten finden sich im Jahr 2020 einige wenige Frauen.

Tabelle 3: Entwicklung Parteizugehörigkeit der Landeshauptleute und Frauenanteil

\begin{tabular}{|c|c|c|c|c|c|c|c|}
\hline Partei & Jahr & $\begin{array}{c}\text { Parteianteil } \\
\text { Landes- } \\
\text { hauptleute }\end{array}$ & $\begin{array}{r}\text { Frauenanteil } \\
\text { Landes- } \\
\text { hauptleute }\end{array}$ & $\begin{array}{r}\text { Parteianteil } \\
\text { Landes- } \\
\text { hauptleute } \\
\text { Stv. }\end{array}$ & $\begin{array}{r}\text { Frauenanteil } \\
\text { Landes- } \\
\text { hauptleute } \\
\text { Stv. }\end{array}$ & $\begin{array}{r}\text { Parteianteil } \\
\text { Landes- } \\
\text { hauptleute } 2 . \\
\text { Stv. }\end{array}$ & $\begin{array}{r}\text { Frauenanteil } \\
\text { Landes- } \\
\text { hauptleute } \\
\text { 2. Stv. }{ }^{* * *}\end{array}$ \\
\hline OEVP & 1990 & 66.67 & 0.00 & 44.44 & 0.00 & 42.86 & 0.00 \\
\hline OEVP & 2000 & 66.67 & 16.67 & 55.56 & 20.00 & 33.33 & 0.00 \\
\hline OEVP & 2010 & 44.44 & 0.00 & 77.78 & 0.00 & NA & NA \\
\hline OEVP & 2020 & 66.67 & 16.67 & 44.44 & 25.00 & 16.67 & 0.00 \\
\hline SPOE & 1990 & 22.22 & 0.00 & 55.56 & 0.00 & 42.86 & 33.33 \\
\hline SPOE & 2000 & 22.22 & 0.00 & 33.33 & 66.67 & 66.67 & 0.00 \\
\hline SPOE & 2010 & 44.44 & 25.00 & 11.11 & 100.00 & 100.00 & 0.00 \\
\hline SPOE & 2020 & 33.33 & 0.00 & 22.22 & 50.00 & 33.33 & 50.00 \\
\hline FPOE & 1990 & 11.11 & 0.00 & NA & NA & 14.29 & 0.00 \\
\hline FPOE & 2000 & 11.11 & 0.00 & 11.11 & 0.00 & NA & NA \\
\hline FPOE & 2020 & NA & NA & 11.11 & 0.00 & 33.33 & 0.00 \\
\hline GRUE* $^{*}$ & 2020 & NA & NA & 22.22 & 50.00 & 16.67 & 100.00 \\
\hline $\mathrm{OTHE}^{* *}$ & 2010 & 11.11 & 0.00 & 11.11 & 0.00 & NA & NA \\
\hline
\end{tabular}

*Stv.: Heinrich Schellhorn seit 2018 in Salzburg, Birgit Hebein von Juni 2019 bis November 2020 in Wien; 2.Stv.: Ingrid Felipe seit 2013 in Tirol

${ }^{* *}$ Gerhard Dörfler von 2009 bis 2013 in Kärnten (FPK, bzw. bis 2009 BZÖ); Stv.: Uwe Scheuch 2009 bis 2012 in Kärnten (ebenfalls FPK, bzw. bis 2009 BZÖ)

${ }^{* * *}$ keinE 2. Stellvertreterln für Landeshauptleute im Burgenland, der Steiermark und Vorarlberg

Quelle: Eigene Berechnungen auf Basis von Daten der Verwaltungshomepages der Bundesländer

Die Analyse des Geschlechterverhältnisses in den österreichischen Landtagen zeigt jedoch, dass der Frauenanteil in der politischen Repräsentation deutlich angestiegen ist. Im Schnitt liegt der Anteil an Frauen im Landtag im Jahr 2020 bei 34 Prozent, und ist damit um 21 Prozent höher als noch vor 30 Jahren. Grafik 1 zeigt einen Überblick der Entwicklung des Anteils der weiblichen Landtagsabgeordneten in den österreichischen Bundesländern. 
Grafik 1: Entwicklung des Anteils der weiblichen Landtagsabgeordneten in den österreichischen Bundesländern

$1990 \quad 2000$
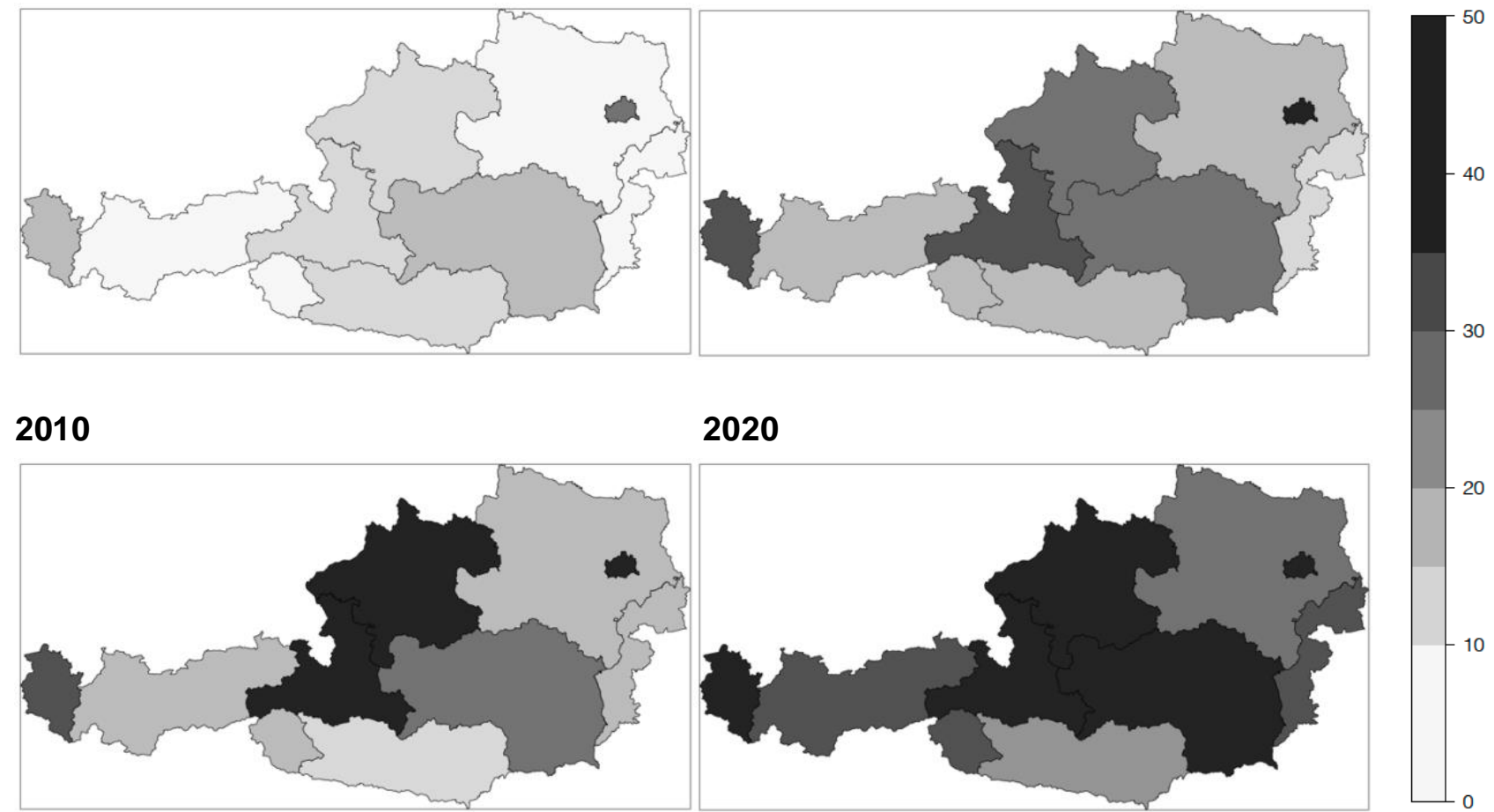

2010

2020
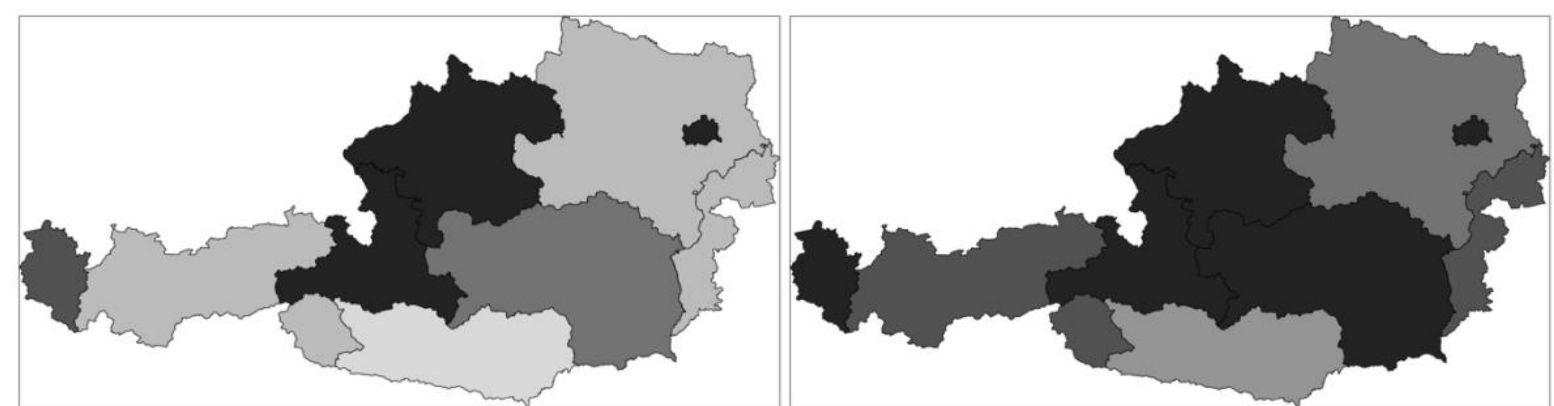

20

Quelle: Eigene Berechnung und Darstellung auf Basis von Daten der Landtagshomepages der Bundesländer

Dieser allgemeine Trend ist nicht nur in den Landtagen, sondern auch in den Landesregierungen deutlich erkennbar. Wie in Grafik 2 dargestellt, steigt der Anteil weiblicher Landesregierungsmitglieder bis ins Jahr 2020 in sechs von neuen Bundesländern über 35 Prozent. 
Grafik 2: Entwicklung des Anteils der weiblichen Landesregierungsmitglieder in den österreichischen Bundesländern

19902000
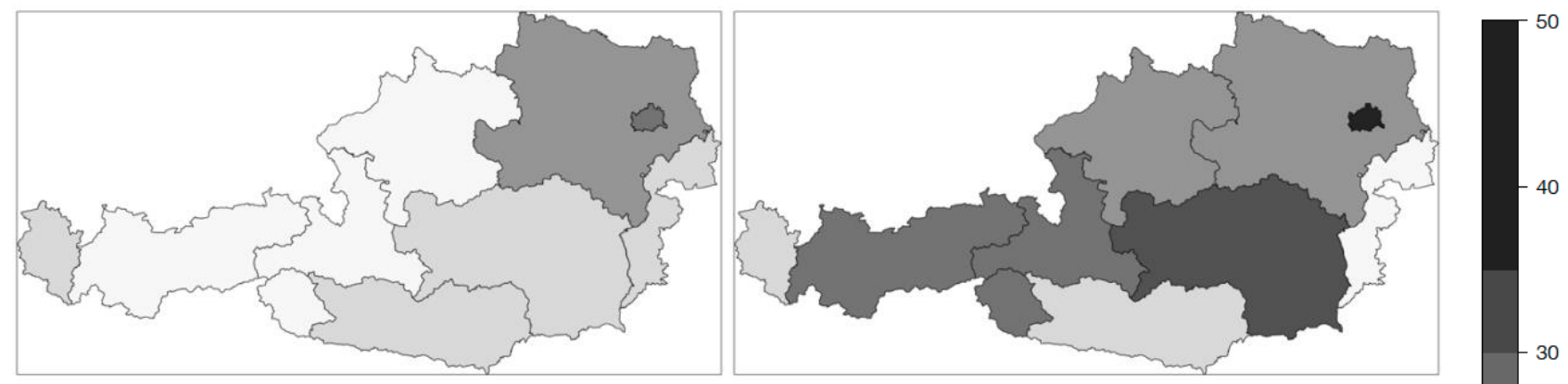

2010

2020
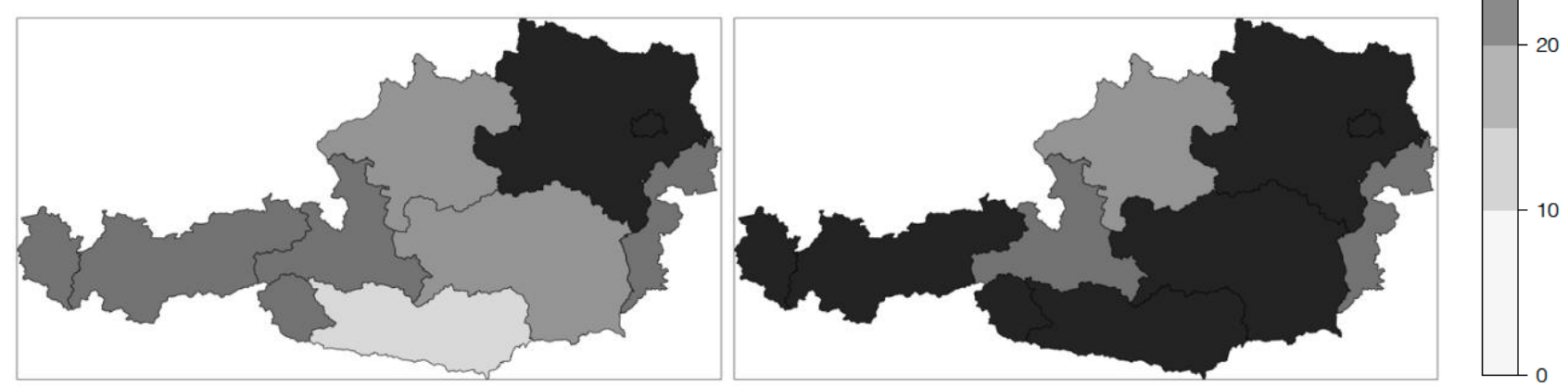

Quelle: Eigene Berechnung und Darstellung auf Basis von Daten der Verwaltungshomepages der Bundesländer

\section{Entwicklung der Politische Repräsentation in den Gemeinden}

In Österreich gibt es aktuell 2095 Gemeinden, und damit 2095 unabhängig tätige Gemeinderäte und Gemeindevorstände, denen jeweils ein Bürgermeister oder eine Bürgermeisterin vorsteht. Stark vereinfacht kann der Gemeinderat mit dem Nationalrat, der Gemeindevorstand mit der Bundesregierung, und das Bürgermeisteramt mit dem Bundeskanzleramt der jeweiligen Gemeinde verglichen werden. Eine wichtige Einschränkung ist jedoch, dass der Gemeinderat anders als der Nationalrat und die Landtage keine gesetzgebende Kompetenz, sondern lediglich Beratungs- und Beschlusskompetenzen besitzt (Anderwald 2013).

Niederösterreich hat mit aktuell 573 am meisten, Vorarlberg mit aktuell 96 am wenigsten unabhängige Gemeinden (vgl. Statistik Austria, Gemeindegrößenklassen mit Einwohnerzahlen). Die durchschnittliche Gemeinde in Österreich hat im Jahr 2020 rund 4250 EinwohnerInnen. Burgenländische Gemeinden haben mit rund $1720 \mathrm{im}$ Schnitt am wenigsten, Salzburger Gemeinden mit rund $4690 \mathrm{im}$ Schnitt am meisten Eihnwohnerlnnen. Durch Zusammenlegung von Gemeinden sind die letzten Jahrzehnte in 
Österreich von einem starken zahlenmäßigen Rückgang der Gemeinden geprägt. Waren es im Jahr 1960 noch 3998, sind es im Jahr 1970 nur mehr 2665 (Anderwald 2013) und seit dem 1. Jänner 2020 lediglich 2095 Gemeinden³.

Die politische Repräsentation der österreichischen Gemeinden wird von allen mindestens 16 Jahre alten, in einer Gemeinde gemeldeten Einwohner Innen mit Hauptwohnsitz direkt gewählt. Anders als auf Landes- und Bundesebene sind auch nicht österreichische EU-BürgerInnen wahlberechtigt ${ }^{4}$. Das Gemeindewahlrecht wird durch die jeweiligen Landesgemeindeordnungen geregelt, welche im Anschluss an die Gemeindeverfassungsnovelle ${ }^{5}$ im Jahr 1962 in Kraft getreten sind. Die Landesgemeindeordnungen unterscheiden sich in Bezug auf die Regelung der politischen Repräsentation in drei wichtigen Punkten. Erstens gibt es unterschiedliche Einwohnerschwellenwerte für die Größe des Gemeinderats. Die Anzahl der Gemeinderatsmitglieder richtet sich zwar in allen Bundesländern nach der Anzahl der Einwohner einer Gemeinde, jedoch ist das durchschnittliche Einwohner-Gemeinderatsmitglied Verhältnis in den Bundesländern sehr unterschiedlich. So sieht etwa die Landesgemeindeordnung im Burgenland für eine Gemeinde mit 1000 Einwohnerlnnen 15 Gemeinderatsmitglieder vor, während die Steirische Landesgemeindeordnung nur 9 vorsieht ${ }^{6}$. Ein zweiter wichtiger Unterschied, ist, dass in Kärnten, Oberösterreich und Tirol nur alle sechs Jahre Wahlen stattfinden, während in den anderen Bundesländern alle fünf Jahre gewählt wird. Außerdem haben Städte mit eigenem Statut (z.B. Innsbruck, St. Pölten, Graz) andere Wahlrythmen als der Rest des jeweiligen Bundeslands. Ein dritter wichtiger Punkt ist die Tatsache, dass BürgermeisterInnen in der Steiermark, Niederösterreich und Wien durch den Gemeinderat gewählt werden, während die anderen Bundesländer schon seit mindestens zwei Jahrzehnten Direktwahlen der durchführen.

Historisch sind Gemeinderatswahlen in Österreich durch eine eher hohe Wahlbeteiligung gekennzeichnet, wobei die Wahlbeteiligung im Westen des Landes und in größeren Gemeinden tendenziell niedriger ist (Stainer-Hämmerle 2013). Auffallend ist jedoch, dass die durchschnittliche Wahlbeteiligung in einigen Bundesländern gesunken ist. So sank zuletzt etwa die Wahlbeteiligung auf

\footnotetext{
${ }^{3}$ vgl. Regionale Gliederung und STATatlas von Statistik Austria:

https://www.statistik.at/atlas/?mapid=topo stadt land\&layerid=layer1 [letzter Zugriff im Mai 2021]

${ }^{4}$ vgl. Österreichs digitales Amt:

https://www.oesterreich.gv.at/themen/leben in oesterreich/wahlen/5/Seite.320621.html\#Aktive [letzter Zugriff im Mai 2021]

${ }^{5}$ zur historischen Ausgangslage, Entstehung und Bewertung der Novelle: https://www.staedtebund.gv.at/oegz/archiv-bis-

2009/details/artikel/die-gemeindeverfassungs-novelle-1962/ [letzter Zugriff im Mai 2021]

${ }^{6}$ siehe Landesgemeindeordnungen der Bundesländer: https://www.ris.bka.gv.at/Land// [letzter Zugriff im Mai 2021]
} 
Gemeindeebene in der Steiermark im Schnitt um zehn, in Vorarlberg um fünf, und in Kärnten um vier Prozentpunkte?

Die in den österreichischen Gemeinderatswahlen gewählte politische Repräsentation unterscheidet sich sowohl in punkto Frauenbeteiligung, also auch was die Verteilung der Mandate auf die Parteien betrifft eindeutig von der Bundes- und Landesebene. Der Frauenanteil unter den BürgermeisterInnen ist in Österreich, nicht nur im Vergleich mit anderen politischen Ebenen, sondern auch verglichen mit anderen Ländern sehr gering. Die vom europäischen Institut für Gleichstellungsfragen gesammelten Daten zeigen, dass Österreich im Jahr 2011 nur knapp fünf Prozent der BürgermeisterInnen Frauen waren. Da der Frauenanteil auch im Jahr 2020 noch unter neuen Prozent liegt, ist Österreich seit 2011 sogar von Platz 25 auf Platz 28 (von 35 untersuchten Ländern) zurückgefallen (EIGE Statistik ${ }^{8}$ ). Die systematische Vollerhebung des Geschlechts der Österreichischen BürgermeisterInnen bestätigt diese Zusammenfassung ${ }^{9}$.

Grafik 3 zeigt die räumliche Verteilung von Männern und Frauen im BürgermeisterInnenamt in den Jahren 2010 und 2018. Die Varianz zwischen den Bundesländern ist allgemein recht gering, und es sind keine geografischen Cluster zu erkennen. Niederösterreich weist im Jahr 2018 als einziges Bundesland einen Frauenanteil von über zehn Prozent auf und hat damit eine fast doppelt so hohe Quote wie die anderen Bundesländer.

\footnotetext{
${ }^{7}$ Details hierzu auf den Verwaltungshomepages der Länder. Steiermark: https://egov.stmk.gv.at/wah/pub/stdwahl/stdwahlSet.do Vorarlberg: https://apps.vorarlberg.at/wahlen/wahl/GV/GV 2020-0913 Kärnten: https://www.ktn.gv.at/wahlen/grwahl2021/ [letzter Zugriff im Mai 2021]

${ }^{8}$ Siehe Gender Statistics Database: https://eige.europa.eu/genderstatistics/dgs/indicator/wmidm pol parl wmid locpol/datatable [letzter Zugriff im Mai 2021]

${ }^{9}$ Eine Zusatzauswertung der Vornamen aller Bürgermeisterlnnen verdeutlicht die die Lage: Im Jahr 2018 gibt es immer noch mehr Josefs (171) als Frauen (164) in Amt. Und es gibt nicht nur 171 Josefs, sondern auch jeweils fast genauso viele mit dem Namen Franz oder Johann.
} 
Grafik 3: Geschlecht der österreichischen BürgermeisterInnen in den Jahren 2010 und 2018
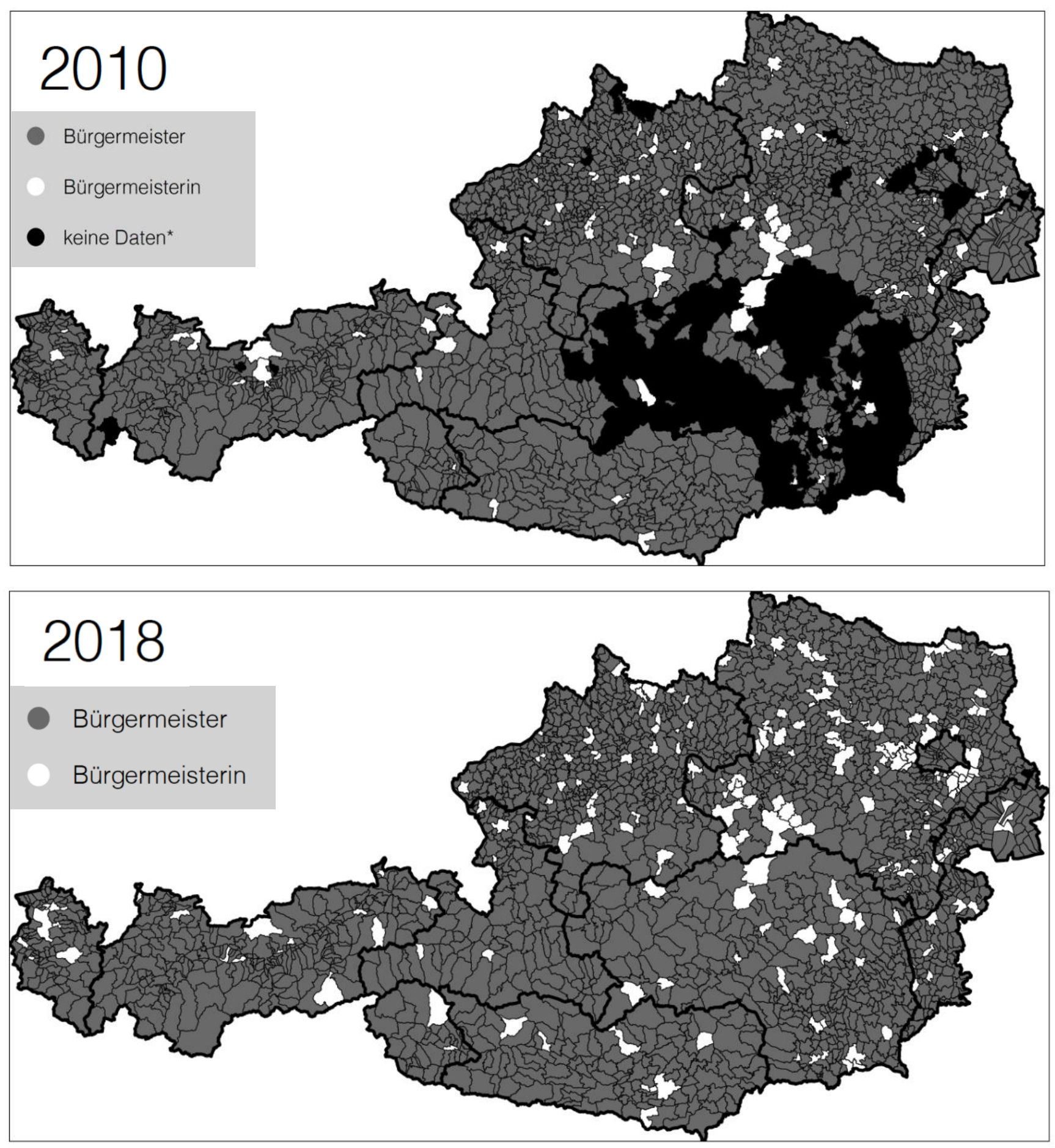

Quelle: Eigene Darstellung auf Basis von Daten der Landesstatistikstellen und dem österreichischen Gemeindebund

Ähnliches wie für das BürgermeisterInnenamt gilt auch für die Frauenquote in den österreichischen Gemeinderäten. Die vom europäischen Institut für Gleichstellungsfragen gesammelten Daten zeigen auch hier, dass Österreich eines der Schlusslichter unter den 36 untersuchten Ländern ist. Im Jahr 2011 ist Österreich mit einem Schnitt von knapp 22 Prozent Frauen im Gemeinderat noch auf Rang 23, 
während es im Jahr 2020 mit nur einem Prozentpunkt mehr auf Platz 32 hinter Serbien, Ungarn, Albanien und einigen weiteren Ländern zurückgefallen ist (EIGE Statistik). Grafik 4 zeigt die die Frauenanteile in den Gemeinderäten Burgenlands, Vorarlbergs, Oberösterreichs, Wiens und der Steiermark, und damit die exakten Daten für etwa die Hälfte der österreichischen Gemeinden. Obwohl detaillierte Daten nicht in allen Bundesländern zugänglich sind, wird der Status Quo sehr gut abgebildet. Insgesamt ergibt sich für die 988 Gemeinden im Jahr 2018 ein Mittelwert von 22,1 Prozent Frauen im Gemeinderat. In 46 Gemeinden sitzt gar keine Frau im Gemeinderat, während nur in drei Gemeinderäten mehr als 50 Prozent Frauen sind. Der Gruppenmittelwerte betragen in der Steiermark 20,4, in Vorarlberg und im Burgenland 21,6 und in Oberösterreich 23,5 Prozent und unterscheiden sich damit nur geringfügig voneinander. Wien hat mit 37 Prozent den höchsten Frauenanteil im Gemeinderat.

Grafik 4: Frauenanteile in den Gemeinderäten der österreichischen Gemeinden

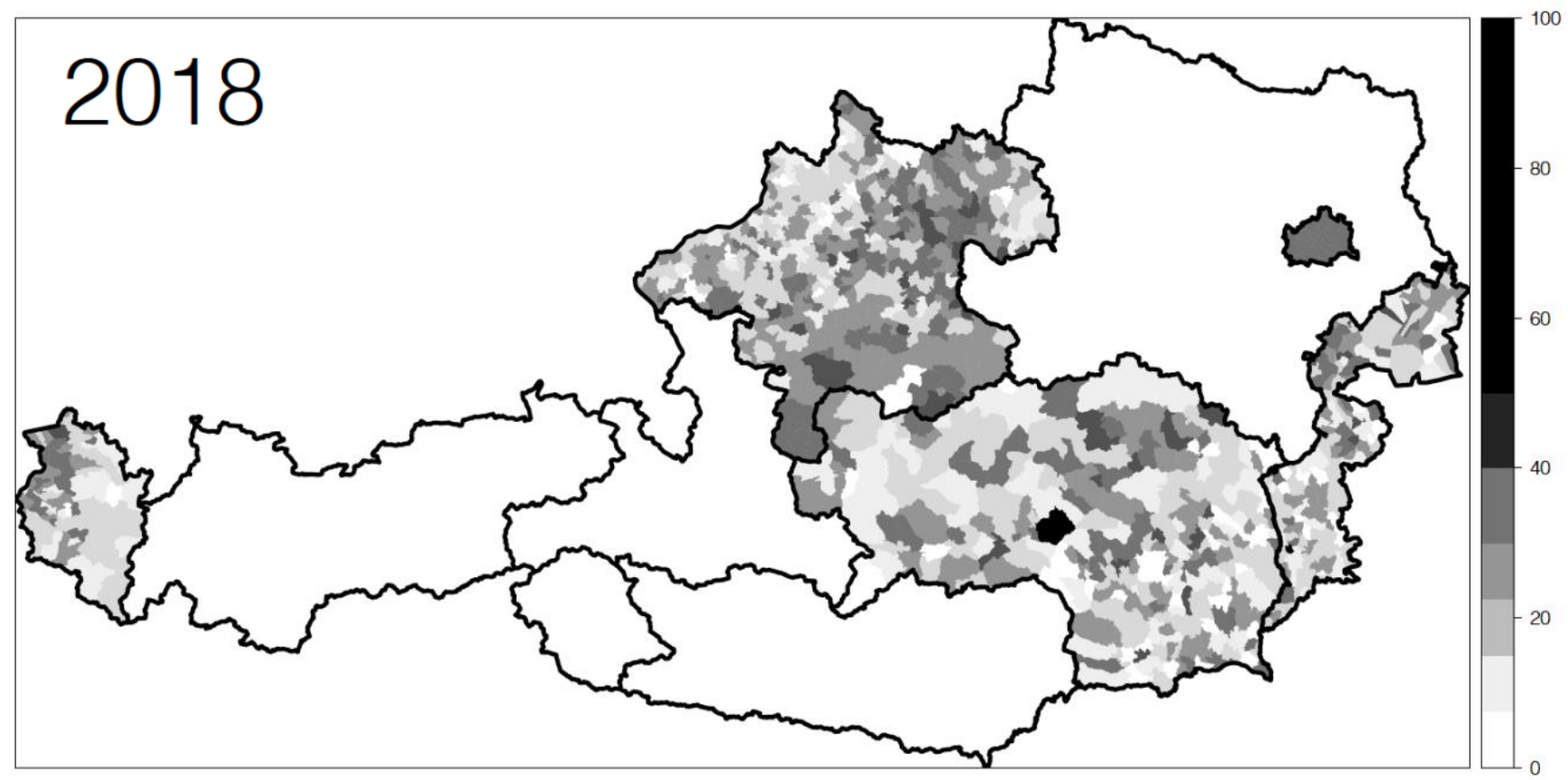

Quelle: Eigene Berechnung und Darstellung auf Basis von Daten der Landesstatistikstellen, Landesregierungsämtern und Landesverwaltungsstellen

Vergleicht man diese Zahlen mit der Landes- und Bundesebene, werden die vertikalen Repräsentationsdifferenzen in Österreich besonders deutlich, wobei auf Gemeindeebene mit Abstand am wenigsten Frauen in politische Ämter gewählt werden. So beträgt im Jahr 2018 der durchschnittliche Frauenanteil im Gemeinderat 22 Prozent, im Landtag 33 Prozent und im Nationalrat 34 Prozent. 
Neben den Frauenanteilen unterschiedet sich auch die durchschnittliche Parteizugehörigkeit der Amtsinhaber auf Gemeinde-, Landes-, und Bundesebene. Sichtbar wird das zum Beispiel an der Dominanz der ÖVP im BürgermeisterInnenamt. Grafik 5 zeigt einen Überblick der Parteizugehörigkeit der österreichischen Bürgermeisterlnnen für das Jahr 2018. In diesem Jahr sind 1199 Bürgermeister, und damit 66 Prozent der parteilich zuordenbaren Ortschefs und Chefinnen Mitglied der ÖVP. Rund 25 Prozent lassen sich der SPÖ zuordnen, 2 Prozent der FPÖ und 7 Prozent zu anderen bzw. unabhängigen Listen.

Grafik 5: Parteizugehörigkeit der österreichischen Bürgermeisterlnnen

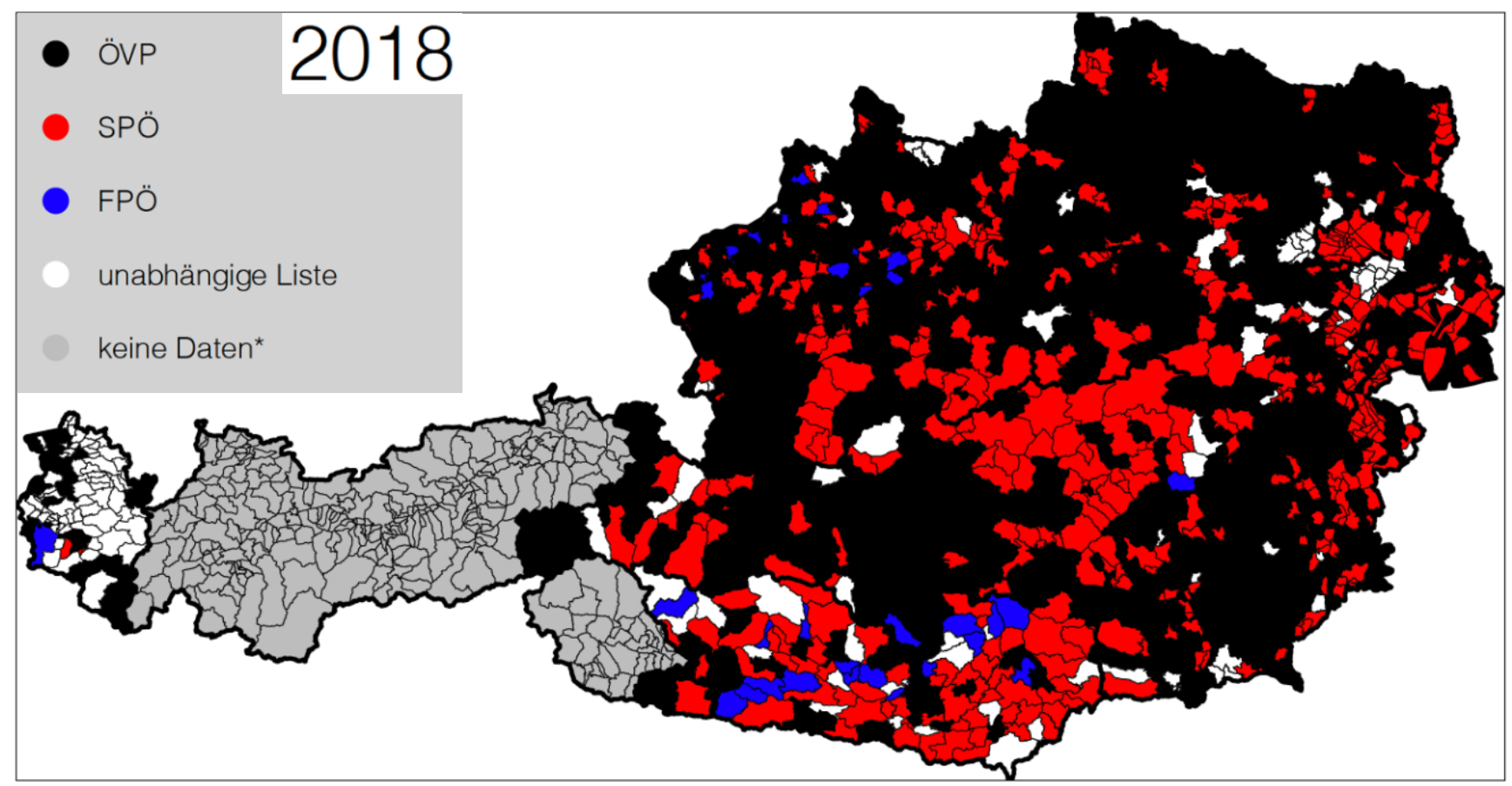

Quelle: Eigene Berechnung und Darstellung auf Basis von Daten der Landesstatistikstellen, Landesregierungsämtern und Landesverwaltungsstellen

Der Grund warum nicht alle 2095 (im Jahr 2018 noch 2096) Bürgermeisterlnnen einer Partei zugeordnet werden können, ist die Tatsache, dass die Tiroler Gemeindewahlordnung aus dem Jahr 1994 keine Parteizugehörigkeit für die Wählbarkeit einer Person vorsieht oder voraussetzt ${ }^{10}$. Aus diesem Grund stellen sich in den Tiroler Gemeinden mehrheitlich Namenslisten der Wahl, welche keine Parteizuordnung ermöglichen. Auf Nachfrage bei den Landesgeschäftsstellen der einzelnen Parteien

\footnotetext{
10 Siehe TGWO 1994 § 8: https://www.ris.bka.gv.at/GeltendeFassung.wxe?Abfrage=LrT\&Gesetzesnummer=20000186 [letzter Zugriff im Mai 2021]
} 
wurde mir mit Verweis auf Datenschutzgründe die Information über Parteimitgliedschaft der Tiroler Bürgermeister verwehrt. Diverse Presseberichte lassen aber Rückschlüsse darauf zu, dass auch in den Tiroler Gemeinden die ÖVP, die mit Abstand am stärksten vertretene Partei ist, während die SPÖ mit nur knapp zwei Dutzend Bürgermeisterlnnen weit abgeschlagen auf dem zweiten Platz noch vor der FPÖ und den Grünen liegt ${ }^{11}$.

Die Kräfteverhältnisse der Parteien in den österreichischen Gemeinderäten spiegeln dieses Bild wider. Die ÖVP beansprucht seit Jahrzehnten mehr als die Hälfte der Gemeinderatssitze in Österreich und ist damit unangefochtene Nummer eins. Wie groß die Stabilität ist und wie gering die Veränderung zeigt sich an den konkreten Zahlen: Im Jahr 2003 hält die ÖVP 52,4 Prozent der Gemeinderatssitze, fünf Jahre später 52,6, weitere fünf Jahre später 54,1 und weitere fünf Jahre später 52,8 Prozent. Im Gegensatz dazu nehmen die Anteile der Gemeindesitze der SPÖ im gleichen Zeitraum leicht von Durchschnittlich 37,2 Prozent auf 31,8 Prozent ab, während die Sitzanteile der FPÖ in diesem Zeitraum zunehmen.

\section{Ursachen für und Auswirkungen von Repräsentationsdifferenzen}

Wie die detaillierte Darstellung der Besonderheiten der österreichischen politischen Repräsentation auf Bundesland- sowie Gemeindeebene zeigt, gibt es erhebliche vertikale, sowie horizontale Varianz. Diese Varianz zeigt sich sowohl auf parteipolitischer Ebene als auch auf Ebene des Geschlechtergleichgewichts in der Repräsentation. Es zeigt sich, dass die Parteipluralität abnimmt, und die Dominanz der ÖVP zunimmt, je kleiner die politische Einheit ist. Im Untersuchungszeitraum liegt der ÖVP Sitzanteil im Parlament im Schnitt bei 33, im Landtag bei fast 40 und im Gemeinderat bei 53 Prozent. Nicht nur das Parteien- sondern auch das Geschlechterverhältnis ist auf Bundesebene am ausgewogensten. Im österreichischen Parlament waren seit 1990 im Schnitt 30 Prozent Frauen, im Landtag 25 und im Gemeinderat weniger als 20 Prozent. Hypothese 1 (,Die vertikale Differenz der politischen Repräsentation ist gering, und wird über die Zeit hinweg immer kleiner') kann nur in Bezug auf die Frauenrepräsentation in der Bundes- und Landesrepräsentation bestätigt werden. Die BundLänder Differenz zwischen den Frauenrepräsentationsquoten beträgt im Jahr 1990 noch 11 Prozent,

\footnotetext{
11 Informationen zu Tiroler Gemeinderatswahl 2016: https://www.meinbezirk.at/imst/c-politik/ergebnisse-der-gemeinderatsund-buergermeisterwahl-2016-in-tirol a1650806 [letzter Zugriff im Mai 2021]
} 
und wird in den darauffolgenden Jahren immer geringer. In Bezug auf alle anderen analysierten Aspekte muss Hypothese 1 verworfen werden. Zwar nimmt die durchschnittliche Frauenrepräsentationsquote auch auf Gemeindeebene zu, allerdings in einem viel geringeren Ausmaß. Dies führt im Laufe des Untersuchungszeitraums zu einer immer größeren Differenz zwischen Bund und Ländern auf der einen und den Gemeinden auf der anderen Seite. Auch für die parteipolitische Komponente kann keine Konvergenz über alle drei politischen Ebenen hinweg festgestellt werden. So bleibt etwa der Anteil an ÖVP Mandaten auf Gemeindeebene im Schnitt über den gesamten Untersuchungszeitraum stabil bei über 50 Prozent, während er im Landtag von knapp 50 Prozent auf nur 35 im Jahr 2020 abnimmt. Die Analyse zeigt zudem, dass nicht nur zwischen den politischen Ebenen (vertikal), sondern auch zwischen den Einheiten ein und derselben Ebene (horizontal) große Unterschied für die Variablen Parteipolitik und Geschlechterverhältnis festgestellt werden können. Daher muss Hypothese 2 (,Die horizontale Differenz der politischen Repräsentation ist niedrig und stabil') verworfen werden. Wie in Tabelle 1 und 2 eindeutig erkennbar wird die Standardabweichung der Parteianteile über die Zeit hinweg größer. Auch auf Gemeindeebene können diese divergierenden Entwicklungen festgestellt werden. Auch die Differenzen der Frauenrepräsentationsquoten in den Gemeinden werden über die Zeit hinweg immer größer. Lediglich in Bezug auf die Frauenquoten in der Landesrepräsentation kann Hypothese 2 teilweise bestätigt werden. Hier zeigt sich am Anfang des Untersuchungszeitraums Divergenz, während die Unterschiede zwischen den Ländern seit 2008 wieder kontinuierlich abnehmen.

Diese Besonderheiten sind auf den ersten Blick nicht logisch erklärbar. Basierend auf der Annahme, dass sowohl das nationale Wahlergebnis, also auch das durchschnittliche Wahlergebnis aller Landeswahlen beziehungsweise Gemeindewahlen, die Präferenz aller Bürger widerspiegelt, und die Parteienlandschaft in Österreich weitgehend über das ganze Land hinweg einheitlich ist, würde man davon ausgehen, dass die Sitze und Ämter im Schnitt auf allen Ebenen ähnlich verteilt sind.

Über die Ursachen für diese Repräsentationsdifferenzen gibt es unterschiedliche Mutmaßungen. Ein sehr wichtiger Ansatz ist in der historischen Entwicklung des österreichischen Bundesstaates zu finden. Die Entstehung des politischen System Österreichs nach den Weltkriegen geht auf einen Kompromiss zwischen der Christlichsozialen und Sozialistischen Partei, also den Vorläufern der heutigen ÖVP und SPÖ, zurück. Die Christlichsozialen befürworteten einen stark dezentralen Bundesstaat nach Vorbild der Schweiz, die Sozialistische Partei hingegen wollte ein einheitsstaatliches politisches System für den 
neuen österreichischen Staat (Bußjäger/Schramek 2020; Bischof/Karlhofer 2016; Kim/Mau 2015). Der seitens der ÖVP klar artikulierte Wunsch nach mehr Föderalismus und weniger Einheitsstaat kann somit ein wichtiger Baustein für die Erklärung der Dominanz auf Bundesland- und Gemeindeeben sein. Gleichzeitig ist davon auszugehen, dass die Positionierung der SPÖ für mehr Einheitsstaat und weniger Föderalismus keinen Fokus auf politische Ämter in Ländern und Gemeinden bewirkt und keine gute Wahlwerbung für Regionalwahlen ist.

Auch der Vergleich zwischen den in diesem Beitrag gefundenen vertikalen Repräsentationsdifferenzen von Frauen und dem aktuellen Forschungsstand zu diesem Thema gibt Rätsel auf. Während das Geschlechtergleichgewicht in der politischen Repräsentation in Österreich auf nationaler Ebene am ausgewogensten ist, ist es in den allermeisten Ländern genau umgekehrt (vgl. z.B. EscobarLemmon/Funk 2018; Vengroff et al. 2003). In der wissenschaftlichen Literatur findet man viele Erklärungsansätze für eine höhere Frauenrepräsentationsquote auf lokaler Ebene. Stockemer und Tremblay (2015) beispielsweise argumentieren, dass subnationale Wahlkampagnen nicht so fordernd sind wie nationale, und daher besser mit den Mehrfachbelastungen des weiblichen Alltags vereinbar sind. Außerdem wird argumentiert, dass Frauen sich mehr für lokale politische Ämter interessieren, weil die lokale Politik mehr mit Alltagsproblemen beschäftigt ist, und daher auch mehr mit den tatsächlichen Problem der Menschen in Berührung kommt (Johnson et al. 2003). Da diese in der wissenschaftlichen Literatur gefundenen Zusammenhänge für Österreich nicht richtig zu sein muss auch Hypothese 3 (,Der Anteil an Frauen in der Repräsentation ist auf nationaler Ebene am geringsten") verworfen werden.

Ein Ansatz für die Erklärung der spezifisch österreichischen vertikalen Unterschiede im Geschlechtergleichgewicht der politischen Repräsentation könnte die Tatsache sein, dass die nationale Repräsentation stärker im öffentlichen Fokus steht, und damit auch größerem Konformitätsdruck ausgesetzt ist. Dieser Druck könnte einerseits von der österreichischen Bevölkerung ausgehen, welche zunehmend an der Gleichberechtigung von Mann und Frau interessiert ist. Andererseits könnte aber auch der oft herangezogenen und vielbeachteten Vergleiche zwischen EU-Ländern oder westlichen Staaten und dem damit einhergehenden Wettbewerbsgedanke ein Katalysator für die nationale Frauenrepräsentationsquote in Österreich sein. Offen bleibt jedoch, warum dieser Mechanismus in anderen Ländern nicht ähnlich funktionieren sollte. 
Ähnlich wie bei den diskutierten Ursachen für Repräsentationsdifferenzen muss auch bei den Auswirkungen der Repräsentationsdifferenzen in unterschiedliche Richtungen geschaut werden. Grob können diese wieder in vertikale und horizontale Auswirkungen eingeteilt werden. Die vertikalen Auswirkungen sind jene, die durch die spezifische Kompetenzverteilung zwischen den föderalen Ebenen entstehen. Dies wird am Beispiel des Politikfeldes Familienpolitik deutlich. Wie in vielen anderen Politikfeldern sind in Österreich die Kompetenzen auch im Feld der Familienpolitik auf alle drei föderalen Ebenen aufgeteilt. Während Maßnahmen wie Elternkarenz, Kinderbeihilfe oder Steuererleichterungen für Familien in Österreich vom Bund geregelt werden, wird das öffentliche Kinderbetreuungsangebot von den Ländern und Gemeinden organisiert und bereitgestellt (Obinger et al. 2005). Diese Tatsache kann in Kombination mit den Repräsentationsdifferenzen dazu führen, dass vor allem jene familienpolitischen Maßnahmen ausgebaut werden, die im Kompetenzbereich von politischen RepräsentantInnen liegen, welche ganz generell den Ausbau von familienpolitischen Maßnahmen befürworten. In Österreich führte das Beispielsweise dazu, dass Geldleistungen, Steuersenkungen und Mutterschutzleistungen schon im 20. Jahrhundert erheblich erweitert wurden. Der Ausbau sozialer Dienstleistungen, wie zum Beispiel institutionellen Kinderbetreuungseinrichtungen begann hingegen später und wurde deutlich langsamer vorangetrieben, als in anderen Ländern (Obinger 2010).

Mögliche horizontale Auswirkungen durch Repräsentationsdifferenzen entstehen durch die Heterogenität der Repräsentation auf regionaler Ebene. Dies kann wiederum gut am Beispiel der institutionellen Kinderbetreuung erklärt werden. Wie Grafik 6 veranschaulicht sind die Kinderbetreuungsquoten für null bis fünfjährige Kinder österreichweit sehr unterschiedlich. Der österreichweite Mittelwert liegt im Jahr 2018 bei 63 Prozent, das Minimum bei 18 und das Maximum bei 100 Prozent. Wobei es einige wenige Gemeinden gibt, für die eine Betreuungsquote von knapp über 100 Prozent berechnet wurde, da die Anzahl der betreuten Kinder, jene der ansässigen Kinder aufgrund von Einpendlern übersteigt. 
Grafik 6: Mögliche Auswirkungen von Repräsentationsidifferenzen: Kinderbetreuungsquoten

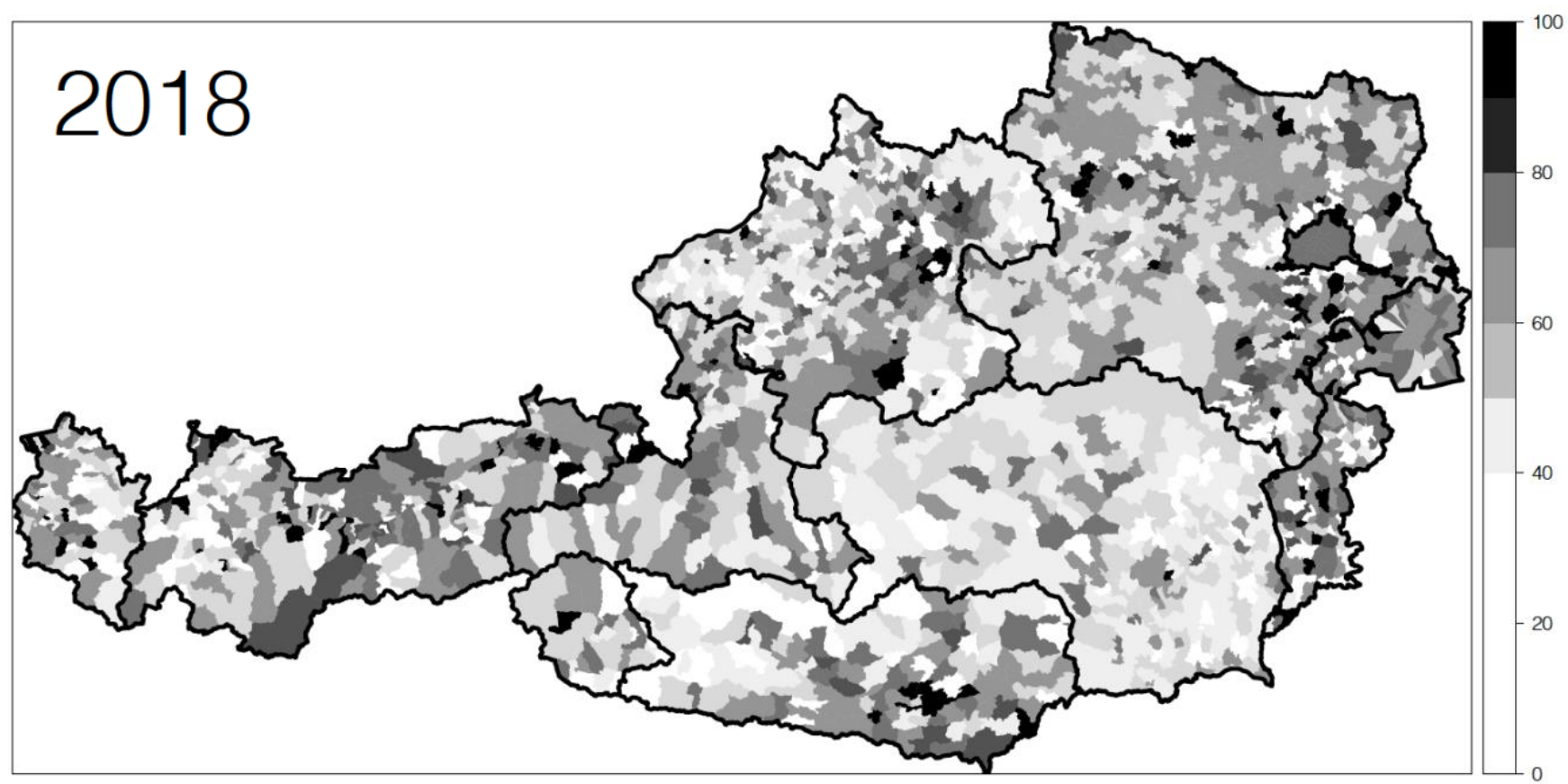

Quelle: Eigene Berechnung und Darstellung auf Basis von Daten der Kindertagesheimstatistik (Daten werden von den Landesstatistikstellen erhoben und von Statistik Austria bereitgestellt)

Wie in Grafik 6 zu sehen können keine eindeutigen geografischen Muster festgestellt werden. Es gibt in allen Bundesländern Gemeinden mit überdurchschnittlich hohen und überdurchschnittlich niedrigen Quoten. Außerdem ist das in vielen wissenschaftlichen Publikationen besprochene Stadt-Land Gefälle (vgl. z.B. Mosimann/Giger 2008; Henning-Smith/Kozhimannil 2016) nicht erkennbar. Ganz anders ist das, wenn man die Verteilung der Ganztagskinderbetreuungsquoten in Österreich betrachtet ${ }^{12}$. Grafik 7 gibt einen Überblick der österreichweit sehr unterschiedlichen Ganztagskinderbetreuungsquoten für null bis fünfjährige Kinder. Der Mittelwert liegt im Jahr 2018 bei 22 Prozent, das Minimum bei null und das Maximum bei einhundert Prozent. Ganz im Unterschied zu den allgemeinen Kinderbetreuungsquoten werden hier eindeutige geografische Muster sichtbar.

\footnotetext{
12 Zwar gibt es keine über alle Bundesländer hinweg einheitliche Definition darüber was „ganztags“ genau bedeutet, aber laut der Dokumentation der Kindertagesheimstatistik „kann bei einer Anwesenheitsdauer von 6 und mehr Stunden pro Tag von "ganztägiger" Anwesenheit ausgegangen werden" (Mayerweck 2020, 23).
} 
Grafik 7: Mögliche Auswirkungen von Repräsentationsidifferenzen: Ganztagskinderbetreuungsquoten

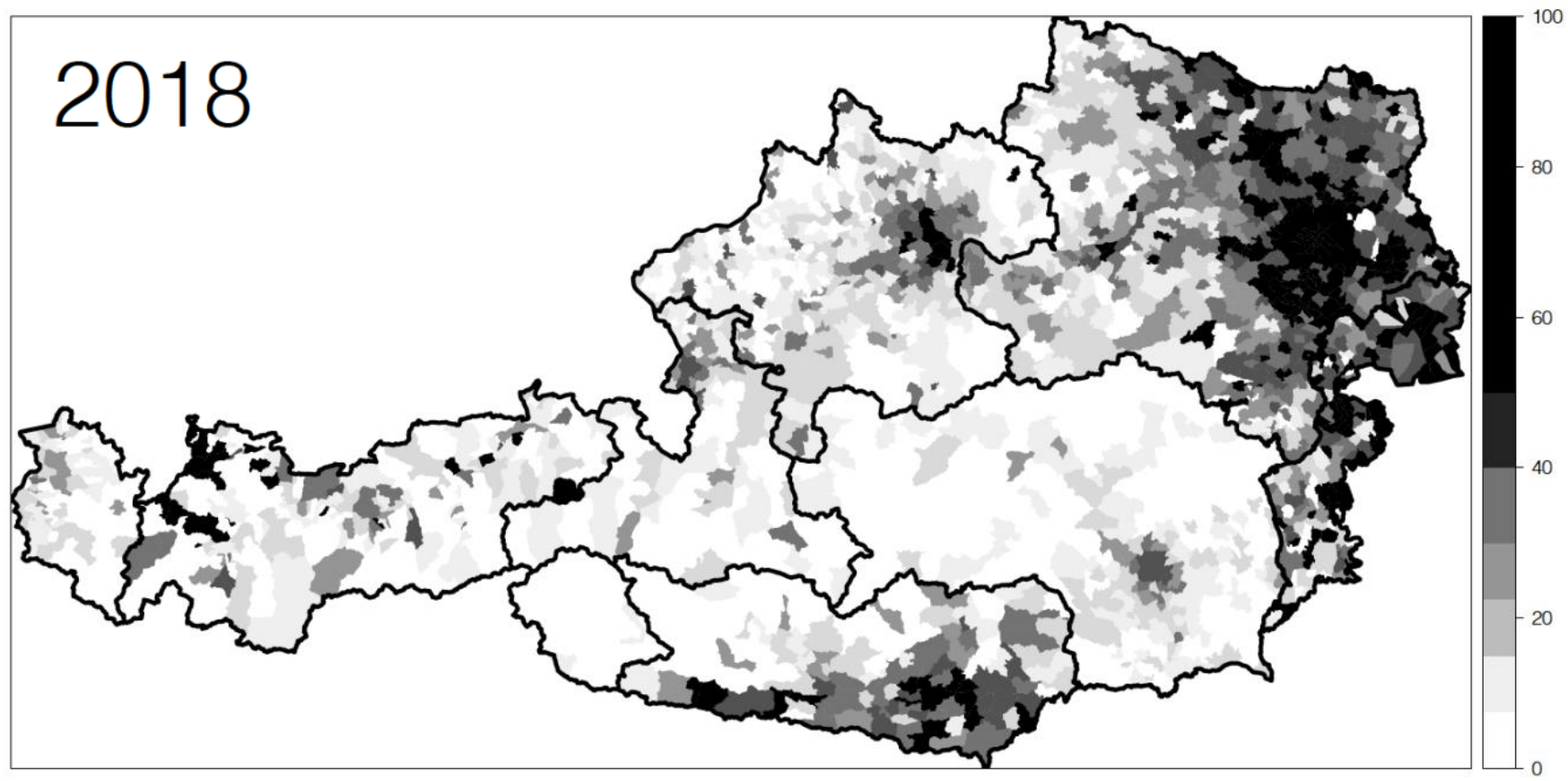

Quelle: Eigene Berechnung und Darstellung auf Basis von Daten der Kindertagesheimstatistik (Daten werden von den Landesstatistikstellen erhoben und von Statistik Austria bereitgestellt)

Der Anteil an Kindern in institutioneller Ganztagsbetreuung ist im Osten des Landes deutlich höher als im Westen. Außerdem sind die Raten in und um größere Städte wie Wien, Graz und Linz deutlich höher als im Rest des Landes. Die im Bereich der Kinderbetreuung auftretende hohe Varianz und die geografischen Muster innerhalb des Landes lassen vermuten, dass es sich nicht um zufällige Entwicklungen oder einen generell ansteigenden Trend handelt, sondern vielmehr um das Ergebnis von politischer Arbeit. Tatsächlich, kommen viele Studien welche auf nationalen Daten basieren zu dem Ergebnis, dass sowohl Parteipolitik (Kittilson 2008; Busemeyer/Seitzl 2018), als auch das Geschlechtergleichgewicht (Bolzendahl 2011; Bonoli/Reber 2010; Ennser-Jedenastik 2017; Bolzendahl/Brooks 2007) einen Einfluss auf das politische Outcome und Output haben. Diese Zusammenhänge auf der Ebene der österreichischen Gemeinden zu erforschen und zu hinterfragen kann aus mindestens drei Gründen von großem wissenschaftlichem Wert sein. Der erste Grund ist die augenscheinlich sehr hohe Varianz des Outcomes (Kinderbetreuungsquoten). Der zweite Grund ist die historische, kulturelle und institutionelle Ähnlichkeit der Analyseeinheiten. Externe Varianz und die Verzerrung aufgrund von ausgelassenen Variablen können dadurch minimiert werden. Ein dritter wichtiger Grund ist die Verfügbarkeit von einer weitaus größeren Fallzahl ( $N=2095)$ im Vergleich zu 
Ländervergleichenden Studien. Die potenzielle Ergiebigkeit der subnationalen österreichischen Repräsentationsdaten sind also Grund genug, um den zeitaufwändigen und mühsamen Prozess des Datensammelns und Datenaufbereitens voranzutreiben und verlässliche Zeitreihen für möglichst viele Repräsentationsvariablen zusammenzutragen.

\section{Zusammenfassung und Diskussion}

Anhand der systematischen statistischen Auswertung von fast 30 Jahren subnationaler politischer Repräsentation veranschaulicht dieser Beitrag, dass sich die nationale und subnationale politische Repräsentation in Österreich stark voneinander unterscheiden und die regionalen Differenzen sehr groß sind. Vertikale Repräsentationsunterschiede zeigen sich durch unterschiedliche Kräfteverhältnisse der Parteien auf den politischen Ebenen. So liegt der ÖVP Sitzanteil im Nationalrat im Schnitt bei 33, im Landtag bei fast 40 und im Gemeinderat bei 53 Prozent.

Nicht nur das Parteien- sondern auch das Geschlechterverhältnis ist auf Bundesebene am ausgewogensten. Im österreichischen Nationalrat waren seit 1990 im Schnitt 30 Prozent Frauen, im Landtag 25 und im Gemeinderat weniger als 20 Prozent.

Nicht nur zwischen den politischen Ebenen, sondern auch zwischen den Einheiten ein und derselben Ebene (horizontal) können große Unterschied festgestellt werden. Während Vorarlberg über viele Jahre hinweg ausschließlich von der ÖVP regiert wurde, regiert im Burgenland seit 2020 ausschließlich die SPÖ. Auch gibt es im Untersuchungszeitraum Gemeinden die ausschließlich von ÖVP, respektive SPÖ regiert werden, sowie Gemeinden, in denen die FPÖ in der Repräsentation dominierend ist. Während die Frauenquote in der Repräsentation auf Bundeslandebene sich zum selben Zeitpunkt um bis zu 32 Prozentpunkte unterscheidet (in den Jahren 1997 und 1998), unterscheidet sie sich auf Gemeindeebene um bis zu 64 Prozent (im Jahr 2018). Interessant ist, dass die maximale Differenz auf Bundeslandebene bis ins Jahr 1997 zugenommen hat, und seither kontinuierlich abnimmt. Auf Gemeindeebene hingegen nimmt die maximale Differenz bis zum letzten Messzeitpunkt immer weiter zu.

Diese empirischen Befunde widersprechen den der aus der Theorie hervorgehenden Erwartungen zu horizontalen und vertikalen Repräsentationsunterschieden in Österreich. Erstens, weil das Geschlechtergleichgewicht in der politischen Repräsentation in Österreich auf nationaler Ebene, und 
nicht wie vermutet auf subnationaler Ebene am ausgewogensten ist. Zweitens, weil die vertikale Differenz der politischen Repräsentation über den Untersuchungszeitraum hinweg nicht geringer geworden ist. Und schließlich drittens, weil die horizontale Differenz der politischen Repräsentation nicht konstant bleibt, sondern im Untersuchungszeitraum größer geworden ist.

Die Ergebnisse und Überlegungen dieses Beitrags zeigen, dass sowohl die Existenz als auch die Ursachen für, und Auswirkungen von horizontalen und vertikalen Repräsentationsunterschieden in föderal organisierten Ländern ein sehr weitreichendes und ergiebiges Forschungsfeld sind. Sie regen eine Diskussion über die Richtung der Entwicklung von politischer Repräsentation und den damit in Zusammenhang stehenden Veränderungsmechanismen an. Sie werfen die Frage auf unter welchen Umständen es in einem Mehrebenensystem zu top-down Veränderungen (wie bei der Frauenrepräsentationsquote in Österreich), und unter welchen Umständen eher zu bottom-up Veränderungen (wie es beispielsweise bei der Frauenrepräsentationsquote in Schweden und Norwegen der Fall zu sein scheint) kommt. Die Ergebnisse dieser Forschung können auch nützlich sein, um die supranationale Ebene (z.B. EU-Wahl Ergebnisse, Entstehung und Entwicklung der EU) besser zu verstehen (Ucakar et al. 2017).

Außerdem sind der ÖVP-SPÖ Föderalismuskompromiss und seine weitreichenden Folgen für die heutigen vertikalen Repräsentationsdifferenzen eine Bereicherung für die Diskussion über die Folgen von historischen Parteidifferenzlinien. Zusätzlich sind die Ergebnisse nützlich, um über die Auswirkungen der Kompetenzverteilung in Föderationen nachzudenken. Was ist Beispielsweise das Ergebnis davon, dass in Österreich die Gemeinden für Kinderbetreuung zuständig sind? Ist die spezifische politische Repräsentation auf Gemeindeebene dafür verantwortlich, dass Österreich in diesem Bereich ein europaweiter Nachzügler ist? Diese und viele weiter Fragen ergeben sich aus der Beschäftigung mit horizontalen und vertikalen Repräsentationsunterschieden. Somit ist dieser Beitrag nicht zuletzt als Appell zu verstehen der subnationalen Forschung, trotz ihrer hohen Komplexität, der Datenverfügbarkeitsproblematik und allgemeiner Generalisierungsskepsis in Zukunft mehr Aufmerksamkeit zu schenken.

\section{Literatur}

Anderwald, Karl (2013). Gemeindepolitik in Österreich, in: Stainer-Hämmerle, Kathrin/Oppitz, Florian 
(Hrsg.): Handbuch Gemeindepolitik, Wien: Verlag Österreich

Bache, lan/Flinders, Matthew V. (Hrsg.) (2004). Multi-level governance, Oxford; New York: Oxford University Press

Behnke, Nathalie/Broschek, Jörg/Sonnicksen, Jared (Hrsg.) (2019). Configurations, Dynamics and Mechanisms of Multilevel Governance, Cham: Springer International Publishing, abrufbar unter: http://link.springer.com/10.1007/978-3-030-05511-0 (letzter Zugriff: 2.2.2021)

Bischof, Günter/Karlhofer, Ferdinand (Hrsg.) (2016). Austrian Studies Today, New Orleans: UNO Press

Bolleyer, Nicole/Bytzek, Evelyn (2009). Government Congruence and Intergovernmental Relations in Federal Systems, in: Regional \& Federal Studies, Vol. 19(3), 371-397

Bolzendahl, Catherine (2011). Beyond the Big Picture: Gender Influences on Disaggregated and Domain-Specific Measures of Social Spending, 1980-1999, in: Politics \& Gender, Vol. 7(01), 35-70

Bolzendahl, C./Brooks, C. (2007). Women's Political Representation and Welfare State Spending in 12 Capitalist Democracies, in: Social Forces, Vol. 85(4), 1509-1534

Bonoli, Giuliano/Reber, Frank (2010). The political economy of childcare in OECD countries: Explaining cross-national variation in spending and coverage rates, in: European Journal of Political Research, Vol. 49(1), 97-118

Busemeyer, Marius R./Seitzl, Lina (2018). The partisan politics of early childhood education in the German Länder, in: Journal of Public Policy, Vol. 38(2), 243-274

Bussjäger, Peter (2018). Föderalismus und Regionalismus in Österreich, in: Bundeszentrale für politische Bildung, 12

Bußjäger, Peter/Schramek, Christoph (2020). Föderalismus und Verfassung, in: Hermann, Andrea Tony/Ingruber, Daniela/Perlot, Flooh/Praprotnik, Katrin/et al. (Hrsg.): Regional. National. Föderal. Zur Beziehung politischer Ebenen in Österreich., Wien

Childs, Sarah/Krook, Mona Lena (2009). Analysing Women's Substantive Representation: From Critical Mass to Critical Actors, in: GOVERNMENT AND OPPOSITION, 21

Dachs, Herbert (2006). Politik in Österreich: das Handbuch, Manz

Enderlein, Henrik/Wälti, Sonja/Zürn, Michael (Hrsg.) (2010). Handbook on multi-level governance, Cheltenham, UK; Northampton, MA: Edward Elgar

Ennser-Jedenastik, Laurenz (2017). How Women's Political Representation affects Spending on Family Benefits, in: Journal of Social Policy, Vol. 46(3), 563-581

Erk, Jan (2004). Austria: A Federation without Federalism, in: Publius: The Journal of Federalism, Vol. 34(1), 1-20

Escobar-Lemmon, Maria C./Funk, Kendall D. (2018). Women's Representation in Subnational Governments: Replicating National Institutions or Forging a Unique Path?, Oxford University Press, abrufbar unter:

https://oxford.universitypressscholarship.com/view/10.1093/oso/9780190851224.001.0001/oso9780190851224-chapter-6 (letzter Zugriff: 17.5.2021)

Fallend, Franz (2007). Does Federalism Matter? Comparing Regional Economic Policies in Austria, Belgium, the Netherlands and Sweden, in: , 22

Fallend, Franz (2003). Föderalismus - eine Domäne der Exekutiven?: Die Bund-LänderVerhandlungen über die Länderforderungsprogramme und die Bundesstaatsreform in Österreich seit 1945, in: Der Bund und die Länder („Geschichte der österreichischen Bundesländer von 1945 bis 1995“, Sonderband 10), Wien 2003, 17-68

Hbbs, Douglas A (1977). Political Parties and Macroeconomic Policy, in: , 22

Henning-Smith, Carrie/Kozhimannil, Katy B. (2016). Availability of Child Care in Rural Communities: 
Implications for Workforce Recruitment and Retention, in: Journal of Community Health, Vol. 41(3), 488-493

Hooghe, Liesbet/Schakel, Arjan H./Marks, Gary/Niedzwiecki, Sara/et al. (2016). Measuring regional authority: a postfunctionalist theory of governance. Volume I First edition., Oxford, United Kingdom ; New York: Oxford University Press

Imbeau, Louis M./Petry, Francois/Lamari, Moktar (2001). Left-right party ideology and government policies: A meta-analysis, in: European Journal of Political Research, Vol. 40(1), 1-29

Johnson, Deb/Kabuchu, Hope/Kayonga, Santa Vusiya (2003). Women in Ugandan local government: the impact of affirmative action, in: Gender \& Development, Vol. 11(3), 8-18

Karlhofer, Ferdinand (2013). Strength through Weakness: State Executive Power and Federal Reform in Austria, in: Swiss Political Science Review, Vol. 19, 19

Kim, Junghun/Mau, Niels Jørgen (2015). Decentralisation of education, health and social protection: issues and challenges - The Copenhagen Workshop 2015, in: , 285

Kittilson, Miki Caul (2008). Representing Women: The Adoption of Family Leave in Comparative Perspective, in: The Journal of Politics, Vol. 70(2), 323-334

Ladner, Andreas (2016). Measuring Local Autonomy in 39 Countries (1990-2014), in: Regional \& Federal Studies, 39

Mayerweck, Elisabeth (2020). Kindertagesheimstatistik 2019/20, in: , 25

Mosimann, Andrea/Giger, Nathalie (2008). Zwischen Parteipolitik und gesellschaftlicher Notwendigkeit. Familienergänzende Kinderbetreuung auf kommunaler Ebene, in: Soziale Welt, Vol. 59(3), 227-246

Obinger, Herbert (2010). Transformations of the welfare state: small states, big lessons 1. publ., Oxford UnivPress, abrufbar unter: http://media.obvsg.at/AC08146036-1001 (letzter Zugriff: 19.3.2019)

Obinger, Herbert/Leibfried, Stephan/Castles, Francis G (2005). Federalism and the Welfare State: New World and European Experiences, Cambridge University Press

Pierson, Paul (1996). The New Politics of the Welfare State, in: World Politics, Vol. 48(2), 143-179

Pierson, Paul (2001). The New Politics of the Welfare State, Oxford University Press

Stainer-Hämmerle, Kathrin (2013). Kommunalwahlen in den Bundesländern, in: Stainer-Hämmerle, Kathrin/Oppitz, Florian (Hrsg.): Handbuch Gemeindepolitik, Wien: Verlag Österreich

Stockemer, Daniel/Tremblay, Manon (2015). Federalism and Women's Representation: Do Federations have more Women Legislators than Centralized States?, in: Publius: The Journal of Federalism, Vol. 45(4), 605-625

Sundström, Aksel/Stockemer, Daniel (2015). What determines women's political representation at the local level? A fine-grained analysis of the European regions, in: International Journal of Comparative Sociology, Vol. 56(3-4), 254-274

Ucakar, Karl/Gschiegl, Stefan/Jenny, Marcelo (2017). Das politische System Österreichs und die EU 5. Auflage., Wien: Facultas

Vengroff, Richard/Nyiri, Zsolt/Fugiero, Melissa (2003). Electoral System and Gender Representation in Sub-National Legislatures: Is There a National---Sub-National Gender Gap?, in: , 12 